For diseases which are often lethal the outcome of a study may be expressed as case-fatality or survival rates. Case-fatality rates (the proportion of episodes of illness which end fatally) express the short-term outcome of disease, but must be interpreted with caution. The definition of an episode of illness is not a specific period of time. Often it refers to a period of medical care, as in a coronary care unit, and case-fatality rates may therefore be altered merely by varying the length of stay in hospital. To measure outcome over longer periods, survival rates are used. These show the proportion of patients surviving over a specified time from the date of diagnosis or the start of treatment. Survival rates may be corrected to allow for deaths from causes other than the disease being studied. As an alternative to survival rates, life-table analyses may be used. An example is shown in the table. These analyses are more revealing than survival rates over one specified period, and are being used increasingly.

\section{Record linkage}

If the various patient records which are routinely compiled were to be linked together this would give a fuller picture of the course of an illness, and of different illnesses occurring in the life of an individual. One simple development of record linkage is matching hospital discharge and mortality data. Much needed information on life expectation after lethal disorders such as myocardial infarction at once becomes available.

There are many potential benefits in more complex linkages, for example between industrial employment and subsequent general practitioner and hospital consultations, but, because of the resources required and the problems of confidentiality, it seems unlikely that there will be much extension of record linkage in the foreseeable future.

\section{The precursor and preclinical phases}

Much current medical practice is concerned with patients in the late stages of chronic and incurable disease. A natural response to this is to identify these illnesses before they become clinically apparent, in the hope of identifying preventable or treatable antecedents. A simple but useful model of a chronic disease divides its evolution into three phases. In the precursor phase abnormalities-for example a raised serum cholesterol concentration-are present without recognisable disease; in the preclinical phase the disease is established, but asymptomatic; in the clinical phase it is overt.

Identifying preclinical disease is the basis of screening and will be discussed in the next article. Among studies of the precursor phase the Framingham study is one of the best known. Some 5000 people aged 30 to 59 were examined annually over a 20-year period and initial concentrations of serum cholesterol and other measurements were correlated with subsequent morbidity and mortality. Among the important findings of this study have been recent observations on the inverse relation between ischaemic heart disease and concentrations of high density lipoprotein cholesterol.

There have been many similar studies. A recent one in Britain consisted of serial measurement of the forced expiratory volume (FEV 1.0) in 800 men over an eight-year period. The lack of correlation between sputum production and the rate of decline of FEV led to the concept of chronic bronchitis as two conditions -an obstructive and a hypersecretory disorder-which frequently coincide but are not causally identical. Studies such as these are an essential part of our attempts to arrest and prevent chronic disease.

Eventually this series will be collected into a book and hence no reprints will be available from the authors.
Occasionally on the wards and in the casualty department I am asked to prescribe sedation in single doses for the distraught relatives of the recently deceased. I doubt both the wisdom and the efficacy of such treatment but pressure from nursing staff and others is great so I usually comply. Would I do better to refuse?

When prescribing in this way it is sometimes difficult to decide if one is treating the bereaved, the onlookers, other patients, the nurses, or oneself. The bereaved should be encouraged to express their sorrow fully, but the effect of the prolonged expression of grief or anguish on the sufferer and on those around is profound. If the doctor can spend an hour or so with the bereaved helping them to work through (and express) their grief all well and good. Possibly a smaller dose of chemical sedative will then be needed. In effect, neither doctor nor nursing staff can devote the necessary time to helping such sufferers. The only alternative to be considered is whether the bereaved should visit their own general practitioner for help. As few patients feel they can trouble their doctor in this way, refusal to help them at the hospital can only lead to unnecessary suffering and possibly lasting bitterness.

If the relative has to stay at the hospital it would seem proper to give a small single dose of a minor tranquilliser or a single dose of ron-barbiturate night sedative. If the relative has to return home a single dose of night sedative is more appropriate, but caution is advisable. Special inquiry should be made in each case for sensitivity to psychotropic drugs and the patient's use of alcohol, and a record should be taken of the details.

Is there any place for long-term anticoagulant treatment in a 65-year-old woman who had auricular fibrillation probably due to ischaemic heart disease and who subsequently developed hemiplegia, most likely embolic in origin. She made good progress with almost complete recovery after two months' physiotherapy. If not, can DC defibrillation preceded by a few days' full anticoagulation be an alternative treatment, and what is the chance of recurrence of $A F$ after defibrillation as a whole?

A systemic embolus may complicate atrial fibrillation of any aetiology, but intra-atrial clot forms most readily in the presence of mitral valve disease and only rarely when the arrhythmia is due to ischaemic heart disease or is idiopathic. Thus long-term prophylactic anticoagulation is mandatory for patients with rheumatic heart disease and atrial fibrillation, unless strongly contraindicated. When fibrillation occurs in the absence of a mitral valve lesion, anticoagulants are not usually given routinely but should be used if any embolic event is suspected: the risk of recurrence is high when the left atrium is the probable source of clot. Anticoagulants should be used cautiously, if at all, in the acute stage of a hemiplegia lest bleeding should occur into the infarcted area of the brain, but treatment is certainly indicated in this convalescent patient. Cardioversion should be avoided if the left atrium is suspected of harbouring clot, and a few days' anticoagulation would not reduce the risk of disaster if sinus rhythm were restored in these circumstances. Moreover, only about $25_{0}^{\circ}$ of patients remain in sinus rhythm for 12 months after cardioversion for atrial fibrillation. ${ }^{1}$ Most patients are best left in controlled fibrillation with the protection of anticoagulation if necessary.

${ }^{1}$ Bjerkelund, C, and Orning, O M, Acta Medica Scandinavica, 1968, 184, 481.

\section{Corrections}

\section{Changing patterns of resource allocation in a London} teaching district

In the paper by Dr J S Yudkin (28 October, p 1212), the address should have read: 28 Huddleston Road, London N7 OAG.

\section{Cymag powder}

The sole distributors in Britain of Cobalt EDTA (Kelocyanor) (Any Questions, 21 October, $p$ 1141) have told us that they do not provide the cyanide emergency kit described. They distribute only boxes containing $1.6 \cdot 20 \mathrm{ml}$ ampoules of Kelocyanor. The emergency kit may be obtained from Cuxson Gerrard \& Co, Oldbury, W Midlands. 